\title{
Dynamic Parameter Identification of UP6 Robot Manipulator using SFLA
}

\author{
Duc Hoang Nguyen \\ Faculty of Electrical and Electronics Engineering \\ HCMC University of Technology \\ Ho Chi Minh City, Vietnam
}

\begin{abstract}
The paper proposes a method using Shuffled Frog Leaping Algorithm (SFLA) to identify dynamic parameters of MOTOMAN UP6 robot manipulator. In this paper, the physical parameters of UP6 including mass, inertia, frictions of the first three joints will be estimated directly without parameterization. SFLA method is also used to find the optimal excitation trajectories. Simulated results verify the effectiveness of SFLA approach, and show that the proposed method achieves a high accuracy.
\end{abstract}

\section{General Terms}

Algorithms.

\section{Keywords}

Optimization, SFLA, Identification, Manipulator.

\section{INTRODUCTION}

The knowledge of the dynamic parameters of the manipulator play an important role in designing model-based algorithms for controlling robot exactly, the validation of the simulation results and the accuracy of the trajectory planning algorithms. Normally, these parameters will be not supplied by manufactures due to copyright problems or difficulties in measuring due to installing sensor, ... Experimental robot identification is the only efficient way to obtain the accurate dynamic parameters [1-3].

A number of methods have been proposed to identify dynamic parameters of robot. In [2], authors used the least squares (LS) method and the particle swarm optimization (PSO) technique to estimate the distinct inertia parameters of Staubli RX-60 robot. These experimental results show that the estimated inertial parameters predict robot dynamics well. In [3], authors demonstrated the possibility of identification methods including least squares, extended Kalman filter, Adaptive Linear Neuron (Adaline) neural networks, Hopfield recurrent neural networks and genetic algorithms in order to obtain parameters characterizing the industrial robot dynamics, specifically in SCARA robots.

Almost methods used for the parameter identification are based on the parameterization of the robot dynamic model, i.e. minimum number of parameters from which the dynamics can be calculated through a linear model with respect to them (base parameter). In this paper, the author introduces an application of the Shuffled Frog Leaping Algorithm in identifying the dynamic parameters of UP6 manipulator without parameterization.

SFLA is a swam-based optimization method and has been successfully applied to solve various optimization problems [4-9]. In this paper, in addition to using for parameter identification, SFLA is also used to find optimal exciting trajectories.

The remaining paper is organized as follows: Section 2 introduces the UP6 robot manipulator and its dynamics. Section 3 presents overview of the Shuffled Frog Leaping Algorithm. The description how to identify the parameters of UP6 robot is given in section 4. Section 5 shows the obtained results and Section 6 concludes this paper.

\section{DYNAMICS OF UP6 MANIPULATOR}

The MOTOMAN UP6 is a high-speed, compact robot that is designed to excel at arc welding, mig welding, and tig welding. However, it can also be utilized for plasma cutting, material handling, and material removal applications. Fig 1. is the picture of the MOTOMAN UP6 robot used in this paper [10].

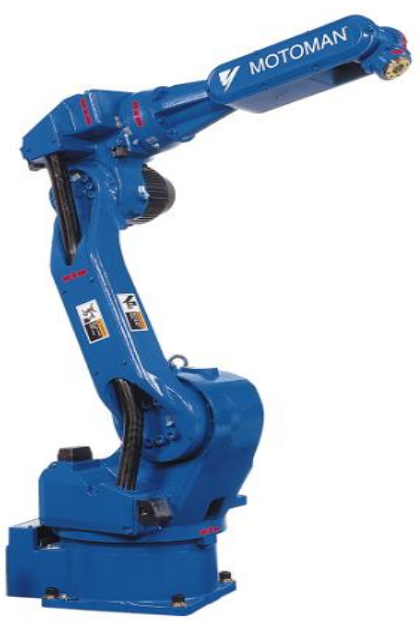

Fig. 1: MOTOMAN UP6 Manipulator (YASKAWA)

The dynamic equations of UP6 robot can be derived from the Newton-Euler formulation. It is commonly written in the matrix form as follows:

$$
D(q) \ddot{q}+H(q, \dot{q}) \dot{q}+G(q)+\tau_{\text {fric }}=\tau
$$

Where $D$ is inertial matrix, $H$ is Coriolis-Centrifugal matrix, $G$ is gravity vector, $\tau$ is applied torque and $\tau_{\text {fric }}$ is friction vectors.

For simplicity, the following friction model is used:

$$
\tau_{\text {fric }}=F_{c} \operatorname{sign}(\dot{q})+F_{v} \dot{q}
$$

Where $F_{c}$ is coulomb friction diagonal matrix and $F_{v}$ is viscous friction diagonal matrix. 
The dynamics of the first three joints of UP6 robot can be modeled by:

$$
\begin{gathered}
{\left[\begin{array}{l}
\tau_{1} \\
\tau_{2} \\
\tau_{3}
\end{array}\right]=\left[\begin{array}{lll}
d_{11} & d_{12} & d_{13} \\
d_{21} & d_{22} & d_{23} \\
d_{31} & d_{32} & d_{33}
\end{array}\right] \ddot{q}+\left[\begin{array}{l}
h_{1} \\
h_{2} \\
h_{3}
\end{array}\right]+\left[\begin{array}{ccc}
f_{v 1} & 0 & 0 \\
0 & f_{v 2} & 0 \\
0 & 0 & f_{v 3}
\end{array}\right] \dot{q}+} \\
{\left[\begin{array}{ccc}
f_{c 1} & 0 & 0 \\
0 & f_{c 2} & 0 \\
0 & 0 & f_{c 3}
\end{array}\right] \operatorname{sign}(\dot{q})+\left[\begin{array}{l}
g_{1} \\
g_{2} \\
g_{3}
\end{array}\right]}
\end{gathered}
$$

Detail about $d_{i j}$ and $h_{i}$ parameters are very long and not given in this paper.

Parameterization of (1) as:

$$
\tau=Y(q, \dot{q}, \ddot{q}) \alpha
$$

Where $Y$ is the linear regressor and $\alpha$ is the set of the base parameters. They can be the combinations of the mass, inertia, friction and gravity parameters [11, 15]. These base parameters can be identified using the least squares method:

$$
\alpha=\left(Y^{T} Y\right)^{-1} Y^{T} \tau
$$

The goal of this paper is to present the SFLA method which can estimate the values of the actual physical parameters (i.e. mass, inertia parameters, friction parameters, etc.) in (3) rather than their combinations in (4), without getting involved with the parameterization procedure.

\section{SHUFFLED FROG LEAPING ALGORITHM (SFLA)}

The SFLA is a meta-heuristic optimization method that mimics the memetic evolution of a group of frogs when seeking for the location that has the maximum amount of available food as illustrated in Fig 2 .

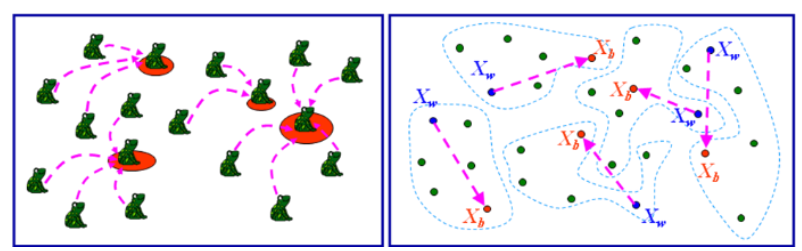

Fig. 2: Evolutionary process of frogs

The algorithm contains the elements of the local search and global information exchange. The SFLA involves a population of the possible solutions defined by a set of virtual frogs that is partitioned into subsets referred to as memeplexes. Within each memeplex, the individual frog holds ideas that can be influenced by the ideas of other frogs, and the ideas can evolve through a process of the memetic evolution. The SFLA performs simultaneously an independent local search in each memeplex using a particle swarm optimization-like method. To ensure global exploration, after a defined number of memeplex evolution steps (i.e. local search iterations), the virtual frogs are shuffled and reorganized into new memeplexes in a technique similar to that used in the shuffled complex evolution algorithm.

In addition, to provide the opportunity for random generation of the improved information, random virtual frogs are generated and substituted in the population if the local search cannot find better solutions. The local searches and the shuffling processes continue until defined convergence criteria are satisfied. The flowchart of the SFLA is illustrated in Fig 3.

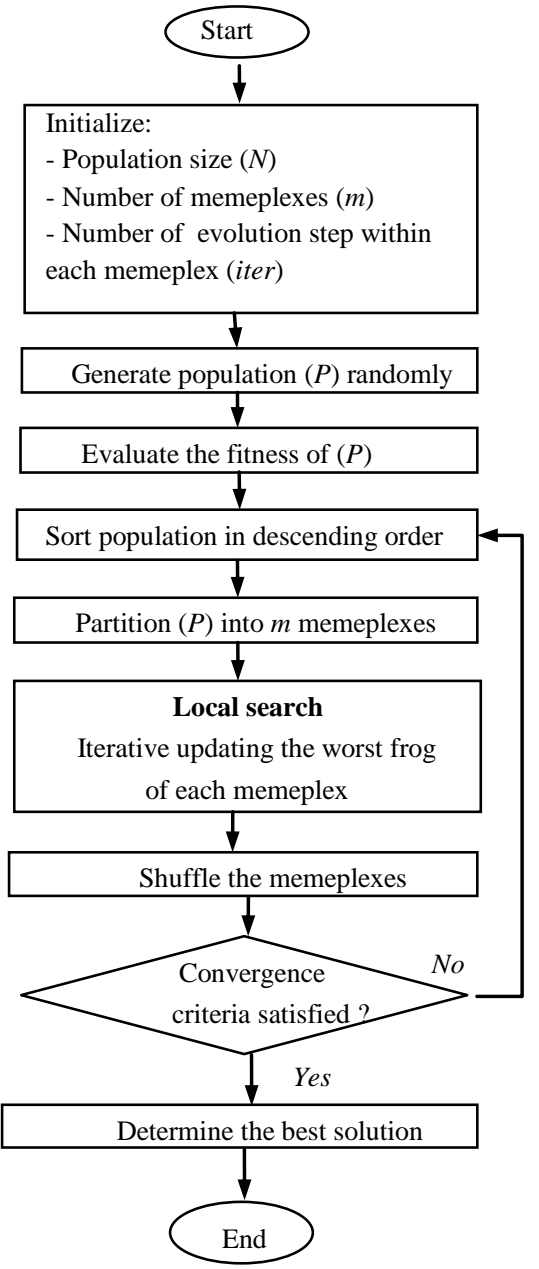

Fig. 3. Flowchart of the SFLA

The idea updating frog leaping rule which is expressed as:

$$
\begin{gathered}
D=\operatorname{r.c}\left(X_{b}-X_{w}\right) \\
X_{w}(\text { new })=X_{w}+D,\|D\| \leq D_{\max }
\end{gathered}
$$

Where $X_{b}$ and $X_{w}$ are identified as the frogs with the best and the worst fitness, respectively; $r$ is a random number between 0 and $1 ; c$ is a constant chosen in the range between 1 and 2 . Refer to [12] for more information.

\section{PARAMETER IDENTIFICATION}

This section discusses the procedures for identifying the parameters of UP6 robot as well as finding the optimal exciting trajectories.

\subsection{Exciting trajectory}

The problem of finding exciting trajectories for the identification has been discussed in several papers. In [13], authors used a genetic algorithm to determine excitation trajectories that minimize either the condition number of the regression matrix or the logarithmic determinant of the Fisher information matrix. In [14-15], authors used modified Fourier series (MFS) as exciting trajectories. The coefficients of MFS are optimized according to the condition number criterion to minimize the sensitivity to measurement noise [14]. 
In [15], Hadamard's inequality is used to propose a novel, simple and intuitive criterion for optimizing an excitation trajectory used for dynamic parameter identification.

In this work, procedure for obtaining the desired trajectory is performed as in the paper [11]. Particularly, exciting trajectory has the following form:

$$
q_{i}(t)=\sum_{k=1}^{N} a_{i k} \sin \left(\omega_{i k} t\right)
$$

Where $\mathrm{N}$ is the degree of the robot, $a_{i k}$ and $\omega_{i k}$ are the parameters need to be identified using SFLA.

\subsection{Parameter Identification using SFLA}

Parameter identification process using SFLA is shown in Fig. 4. As can be seen from the figure, SFLA is used to minimize error between the measured torque and the estimated torque.

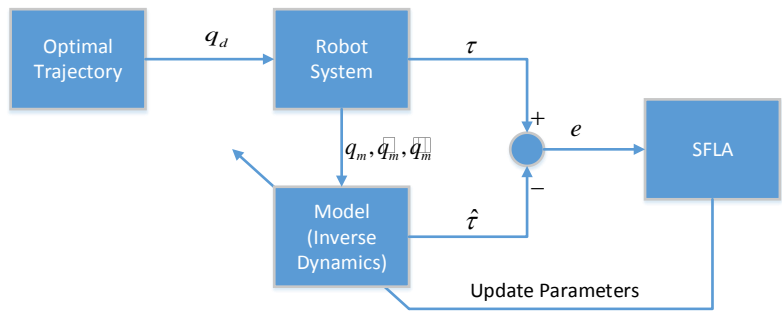

Fig. 4: Identification process using SFLA

The estimated torque is calculated from the inverse dynamics model as follows:

$$
\hat{\tau}=\widehat{D}(q) \ddot{q}+\widehat{H}(q, \dot{q}) \dot{q}+G(q)+\hat{\tau}_{\text {fric }}
$$

Where $\widehat{D}, \widehat{H}$ and $\hat{\tau}$ are calculated according to the estimated parameters.

The procedure for minimizing the error $e$ using SFLA as follows [11]:

- Collecting $\mathrm{N}$ data samples including torque, position, velocity and acceleration of each joint.

- Computing the objective function:

$$
f=\|E\|_{2}
$$

Where $E=\left|\begin{array}{llll}e_{1} & e_{2} & \cdots & e_{N}\end{array}\right|$

- $\quad$ Run SFLA such that minimizing (10)

\section{SIMULATION RESULTS}

\subsection{Finding the exciting trajectory}

As presented in section 4.1, the exciting trajectories for the first three joints have the following form:

$q_{1}(t)=a_{11} \sin \left(\omega_{11} t\right)+a_{12} \sin \left(\omega_{12} t\right)+a_{13} \sin \left(\omega_{13} t\right)$

$q_{2}(t)=a_{21} \sin \left(\omega_{21} t\right)+a_{22} \sin \left(\omega_{22} t\right)+a_{13} \sin \left(\omega_{23} t\right)$

$q_{3}(t)=a_{31} \sin \left(\omega_{31} t\right)+a_{32} \sin \left(\omega_{32} t\right)+a_{33} \sin \left(\omega_{33} t\right)$

Where $a_{i k}$ and $\omega_{i k}$ with $i, k=1: 3$ need to be identified. In this work, these parameters (18 variables in total) are identified by SFLA. Particularly as follows:
- Computing the samples using formulas (11) at the instants $t=\frac{T}{N} i(i=1: N)$ and arranging them inside $\mathrm{Q}$ as follows:

$$
=\left[\begin{array}{ccccccccc}
q_{11} & \dot{q}_{11} & \ddot{q}_{11} & q_{21} & \dot{q}_{21} & \ddot{q}_{21} & q_{31} & \dot{q}_{31} & \ddot{q}_{31} \\
q_{12} & \dot{q}_{12} & \ddot{q}_{12} & q_{22} & \dot{q}_{22} & \ddot{q}_{22} & q_{32} & \dot{q}_{32} & \ddot{q}_{32} \\
\vdots & \vdots & \vdots & \vdots & \vdots & \vdots & \vdots & \vdots & \vdots \\
q_{1 N} & \dot{q}_{1 N} & \ddot{q}_{1 N} & q_{2 N} & \dot{q}_{2 N} & \ddot{q}_{2 N} & q_{3 N} & \dot{q}_{3 N} & \ddot{q}_{3 N}
\end{array}\right]
$$

With $\mathrm{T}$ is the sampling time and $\mathrm{N}$ is the number of samples; $q_{i j}, \dot{q}_{i j}, \ddot{q}_{i j}$ are the position, velocity and acceleration of the joint $i$ at the $j$ th sample, respectively.

- Computing the objective function as (12).

$$
J=\frac{1}{\left\|Q^{T} Q\right\|_{2}}
$$

With $\|.\|_{2}$ is the 2-norm of the matrix.

- $\quad$ Run SFLA such that minimizing the objective function $J$. Then, we yield parameters $a_{i k}$ and $\omega_{i k}$ and so, the exciting trajectories.

Note that $q_{i j}, \dot{q}_{i j}$ values have to satisfy with the constraints of the UP6 robot:

$$
\begin{gathered}
-170 \mathrm{deg} \leq q_{1} \leq 170 \mathrm{deg},-140 \mathrm{deg} / \mathrm{s} \leq \dot{q}_{1} \leq 140 \mathrm{deg} / \mathrm{s} \\
-90 \mathrm{deg} \leq q_{2} \leq 155 \mathrm{deg},-160 \mathrm{deg} / \mathrm{s} \leq \dot{q}_{2} \leq 160 \mathrm{deg} / \mathrm{s} \\
-170 \mathrm{deg} \leq q_{3} \leq 190 \mathrm{deg},-170 \mathrm{deg} / \mathrm{s} \leq \dot{q}_{3} \leq 170 \mathrm{deg} / \mathrm{s}
\end{gathered}
$$

The above steps are implemented by Matlab software. After running SFLA, the obtained results as Fig. 5. and Table 1.

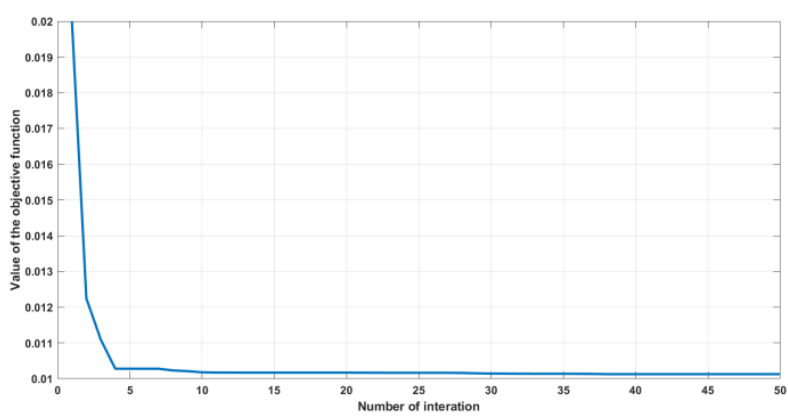

Fig. 5: Evolving process of the objective function $J$

Table 1. The parameters of the exciting trajectories

\begin{tabular}{|c|c|c|c|c|c|c|}
\hline \multirow{2}{*}{$\begin{array}{c}\text { Joint } \\
1\end{array}$} & $a_{11}$ & $a_{12}$ & $a_{13}$ & $\omega_{11}$ & $\omega_{12}$ & $\omega_{13}$ \\
\cline { 2 - 7 } & 0.5946 & 1.1910 & 0.8395 & 1.2728 & 1.2614 & -0.4188 \\
\hline \multirow{2}{*}{$\begin{array}{c}\text { Joint } \\
2\end{array}$} & $a_{21}$ & $a_{22}$ & $a_{23}$ & $\omega_{21}$ & $\omega_{22}$ & $\omega_{23}$ \\
\cline { 2 - 7 } & -0.6776 & 0.4117 & -0.4006 & 1.2666 & -0.0485 & 0.7702 \\
\hline \multirow{2}{*}{$\begin{array}{c}\text { Joint } \\
3\end{array}$} & $a_{31}$ & $a_{32}$ & $a_{33}$ & $\omega_{31}$ & $\omega_{32}$ & $\omega_{33}$ \\
\cline { 2 - 7 } & 1.2101 & 0.8244 & 1.2484 & -1.4820 & -0.1229 & 1.0399 \\
\hline
\end{tabular}

The optimized exciting trajectories of the first three joints are shown in Fig. 6. 


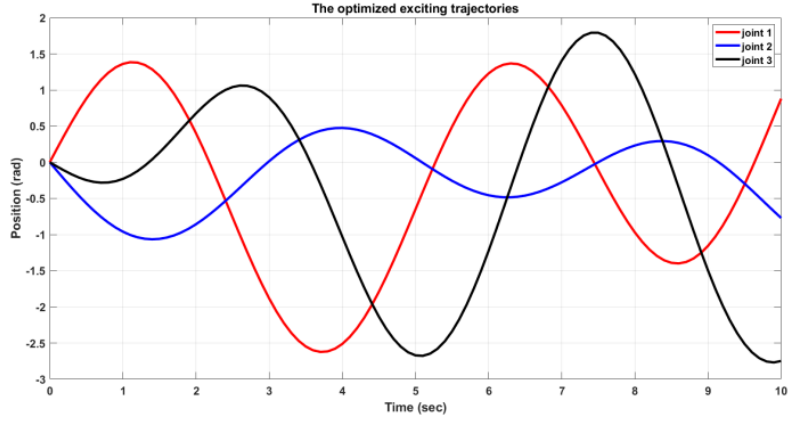

Fig. 6: The optimized exciting trajectories

\subsection{Identifying the parameters of the UP6}

Since the UP6 robot is unstable, controllers need to be designed to stabilize the position of the joints. In this work, three PID controllers are used to stabilize the first three joints of UP6 robot. Fig. 7 and Fig. 8 present the closed loop controlling diagram and PID controllers for the first three joints. PID controllers are designed such that the errors between the measured positions and the desired positions are the possible smallest. Fig. 9 and Fig. 10 are the obtained results when controlling positions by PID controllers. As can be seen from Fig. 10, position errors are rather small. So, these results can be used to collect sample data for identifying the dynamic parameters of UP6 robot.

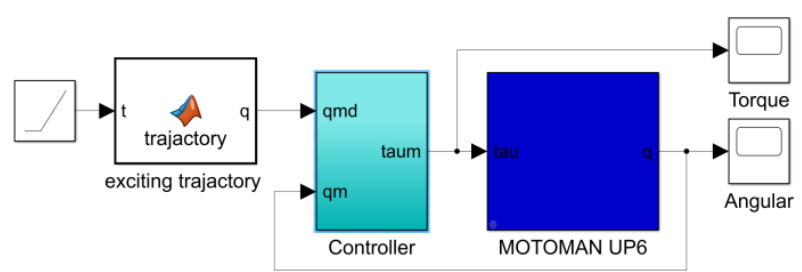

Fig. 7: Simulink schematic controlling the UP6 robot

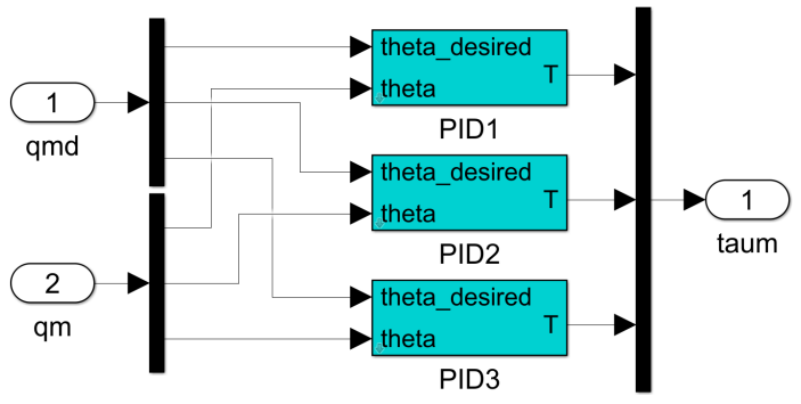

Fig. 8: PID controllers for the first three joints

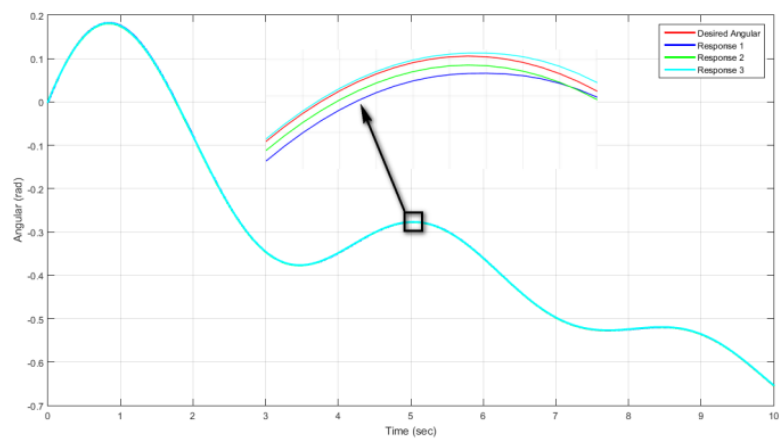

Fig. 9: Closed loop response of the first three joints

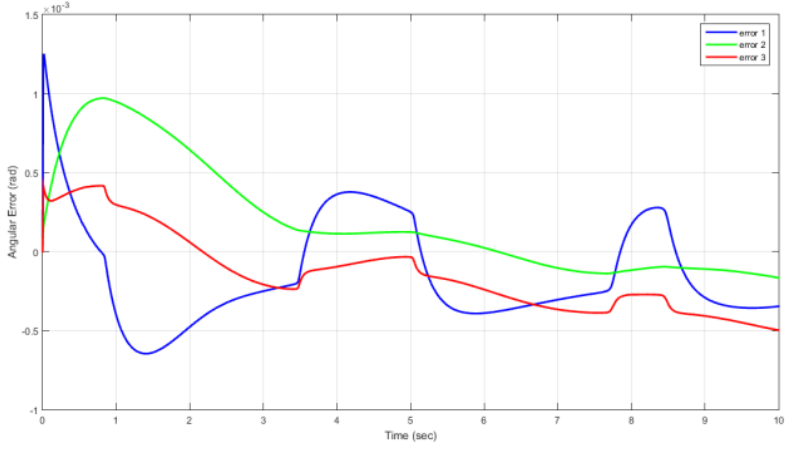

Fig. 10: Position errors of the first three joints

The procedures to identify the dynamic parameters of UP6 robot using SFLA are presented as follows: firstly, the optimized exciting trajectories are used as the reference positions of the PID controllers to control position of the joints. Secondly, torque data is collected along with response position, velocity and acceleration of three joints. These response position, velocity and acceleration data are fed into (9) to get the estimated torque. Then, SFLA is used to minimize the error between the measured torque and the estimated torque according to the formula (10).

The parameter settings of SFLA are shown in Table 2.

Table 2. The SFLA parameter settings

\begin{tabular}{|c|c|c|c|c|c|}
\hline $\mathrm{N}$ & $\mathrm{G}$ & $\mathrm{C}$ & $\mathrm{m}$ & iter & $\mathrm{D}_{\max }$ \\
\hline 50 & 50 & 2 & 10 & 10 & $\infty$ \\
\hline
\end{tabular}

There are many parameters in dynamics model of UP6 robot need to be identified. However, in this paper, the 12 most important parameters of UP6 robot are considered. They are the mass, the inertia, the coulomb friction and the viscous friction of the first three links. The values of these parameters will be identified using SFLA. For simulating purpose, the values of these parameters are given in Table 3 (Column: Virtual values). While the values of the mass of three links are measured by disassembling the links of the robot, the remaining values are simulated.

The estimated values of these 12 parameters after running SFLA are given in Table 3 (Column: Estimated values). As can be seen from Table 3, the estimated values are rather close to the simulated values. To validate the obtained results, the responses of the position of three joints will be compared between the real model and the estimated model.

Table 3. The dynamic parameters of UP6 robot

\begin{tabular}{|l|l|l|}
\hline Parameters & Virtual values & Estimated values \\
\hline Mass of link 1: $\mathrm{m}_{1}$ & 60 & 55.9035 \\
\hline Mass of link 2: $\mathrm{m}_{2}$ & 35 & 35.1481 \\
\hline Mass of link 3: $\mathrm{m}_{3}$ & 35 & 34.9386 \\
\hline Inertia of link 1: $\mathrm{I}_{\mathrm{yy}}$ & 1.2 & 1.2740 \\
\hline Inertia of link 2: $\mathrm{I}_{2 \mathrm{zz}}$ & 0.7 & 0.7077 \\
\hline Inertia of link 3: $\mathrm{I}_{3 \mathrm{vv}}$ & 0.7 & 0.7000 \\
\hline Coulomb friction 1: $\mathrm{f}_{\mathrm{cl}}$ & 15 & 13.5045 \\
\hline Coulomb friction 2: $\mathrm{f}_{\mathrm{c} 2}$ & 5 & 2.2423 \\
\hline Coulomb friction $3: \mathrm{f}_{\mathrm{c} 3}$ & 12 & 14.2434 \\
\hline Viscous friction $1: \mathrm{f}_{\mathrm{v} 1}$ & 29 & 30.0105 \\
\hline Viscous friction $2: \mathrm{f}_{\mathrm{v} 2}$ & 10 & 10.7523 \\
\hline Viscous friction 3: $\mathrm{f}_{\mathrm{v} 3}$ & 15 & 16.1701 \\
\hline
\end{tabular}

Fig. 11, Fig. 12 and Fig. 13 compare the position responses of three joints. It can be seen in the figures that the estimated model give response results very close to the real model. 
Fig. 14, Fig. 15 and Fig. 16 compare the torque responses of three joints. It can be seen in the figures that the estimated model give response results close to the real model.

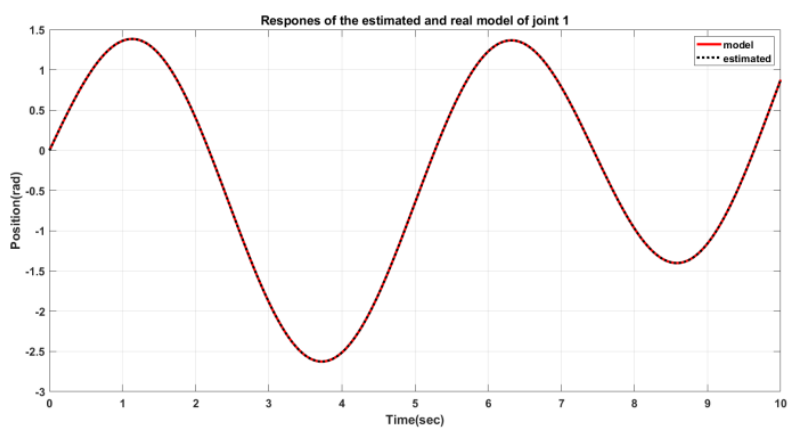

Fig. 11: Position response of the estimated and real model of the joint 1

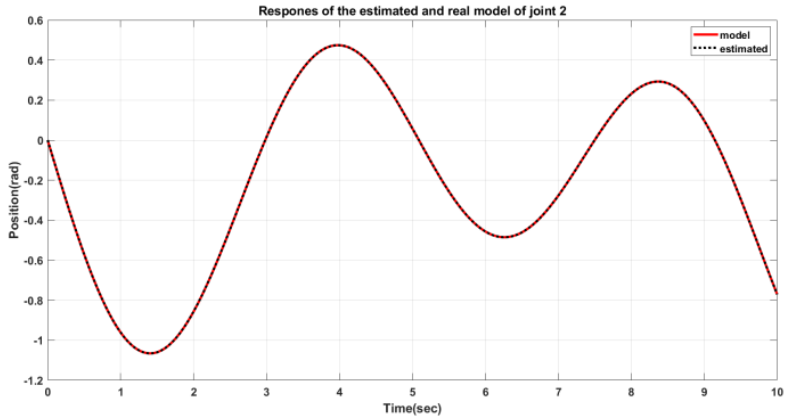

Fig. 12: Position response of the estimated and real model of the joint 2

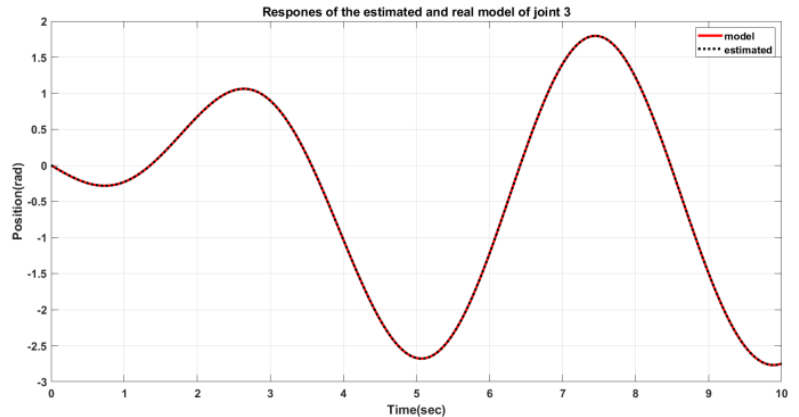

Fig. 13: Position response of the estimated and real model of the joint 3

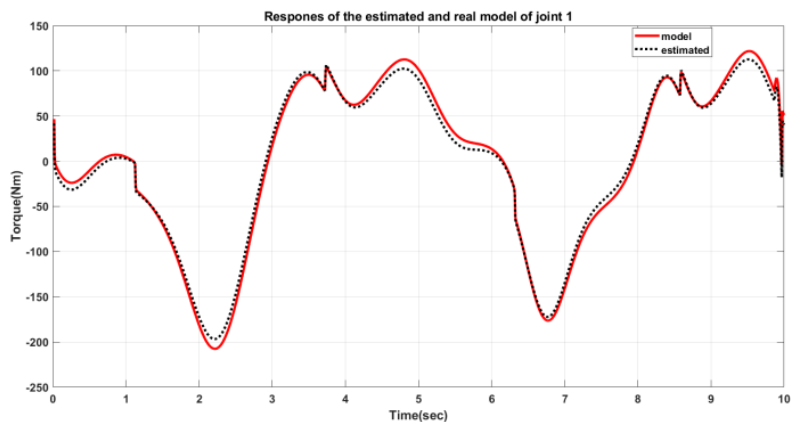

Fig. 14: Torque response of the estimated and real model of the joint 1

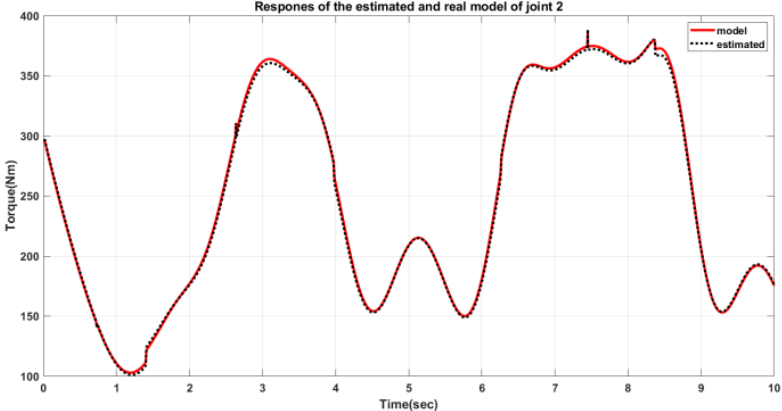

Fig. 15: Torque response of the estimated and real model of the joint 2

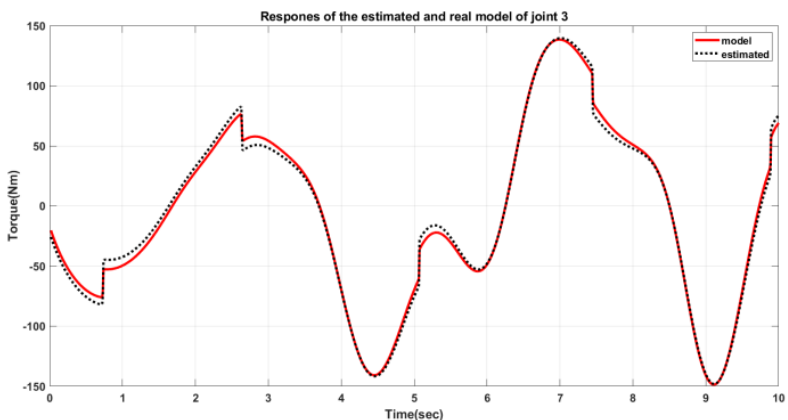

Fig. 16: Torque response of the estimated and real model of the joint 3

To further validate, the root mean square (RMS) of the errors between the estimated and model values will be calculated. The results are presented in Table 4 .

Table 4. The RMS of the errors between the estimated and model values

\begin{tabular}{|l|l|l|l|}
\hline & Joint 1 & Joint 2 & Joint 3 \\
\hline Position & $1.30 \mathrm{e}-4$ & $4.03 \mathrm{e}-6$ & $2.01 \mathrm{e}-5$ \\
\hline Torque & 6.9587 & 2.0493 & 4.2899 \\
\hline
\end{tabular}

The above results show that although the values of the estimated parameters have deviation comparing to virtual values, the responses of the position of joints as well as the torque of the identified model are rather close to the real model.

\section{CONCLUSION}

In this paper, SFLA is applied to identify the dynamic parameters of the UP6 robot manipulator without parameterization, i.e. the physical parameters such as the mass, the inertia, the friction are identified instead of their combinations. The simulated results show that SFLA gives response results close to the real model. In the future, the obtained results of SFLA will be compared to other methods in finding the optimal exciting trajectories and identifying the dynamic parameters of robot manipulator. In addition to, the number of the identified parameters will be also increased. These results will be also apply to the real UP6 manipulator.

\section{REFERENCES}

[1] Basilio Bona and Aldo Curatella. (2005). Identification of Industrial Robot Parameters for Advanced ModelBased Controllers Design. Proceedings of the IEEE, International Conference on Robotics and Automation.

[2] Zafer Bingül, Og uzhan Karahan. (2011). Dynamic identification of Staubli RX-60 robot using PSO and LS 
methods. Expert Systems with Applications 38, 41364149 .

[3] Claudio Urrea, José Pascal. (2016). Design, simulation, comparison and evaluation of parameter identification methods for an industrial robot. Computers and Electrical Engineering, 1-16.

[4] Muzaffar Eusuff, Kevin Lansey and Fayzul Pasha. (2006). Shuffled frog-leaping algorithm: a memetic meta-heuristic for discrete optimization. Engineering Optimization Vol. 38, No. 2, 129-154.

[5] A. Darvishi, A. Alimardani, B. Vahidi, S.H. Hosseinian. (2014). Shuffled Frog-Leaping Algorithm for Control of Selective and Total Harmonic Distortion. Journal of Applied Research and Technology, Volume 12, Issue 1, 111-121.

[6] Kaushik Kumar Bhattacharjee, S.P. Sarmah. (2014). Shuffled frog leaping algorithm and its application to 0/1 knapsack problem. Applied Soft Computing, Volume 19, 252-263.

[7] Yi Han, et al. (2013). Shuffled Frog Leaping Algorithm for Preemptive Project Scheduling Problems with Resource Vacations Based on Patterson Set. Journal of Applied Mathematics.

[8] Daniel Mora-Melia, Pedro L. Iglesias-Rey, F. Javier Martínez-Solano and Pedro Muñoz-Velasco. (2016). The Efficiency of Setting Parameters in a Modified Shuffled Frog Leaping Algorithm Applied to Optimizing Water Distribution Networks. Water 2016, 8, 182, MDPI.
[9] Dina M. Said, Nabil M. Hamed, Almoataz Y. Abdelaziz. (2016). Shuffled Frog Leaping Algorithm for Economic Dispatch with Valve Loading Effect. International Electrical Engineering Journal, Vol 7 No 3.

[10] Industrial Robot MOTOMAN-UP6, MOTOMAN ROBOTICS EUROPE AB.

[11] Hossein Jahandideh, Mehrzad Namvar. (2012). Use of PSO in Parameter Estimation of Robot Dynamics; Part One: No Need for Parameterization .16th International Conference on System Theory, Control and Computing (ICSTCC).

[12] Duc-Hoang Nguyen and Thai-Hoang Huynh. (2008). A SFLA-Based Fuzzy Controller for Balancing a Ball and Beam System. Tenth IEEE International Conference on Control, Automation, Robotics and Vision (ICARCV 2008), Hanoi, Vietnam, 17-20.

[13] G. Calafiore, M. Indri, and B. Bona. (2001). Robot Dynamic Calibration: Optimal Excitation Trajectories and Experimental Parameter Estimation. Journal of Robotic Systems 18(2), 55-68.

[14] Wenxiang $\mathrm{Wu}$, Shiqiang Zhu, Xuanyin Wang and Huashan Liu. (2012). Closed-loop Dynamic Parameter Identification of Robot Manipulators Using Modified Fourier Series. International Journal of Advanced Robotic Systems, Vol. 9, 29.

[15] Jingfu Jin, Nicholas Gans. (2015). Parameter identification for industrial robots with a fast and robust trajectory design approach. Robotics and ComputerIntegrated Manufacturing 31, 21-29. 